\title{
Prospects for control of mosquito-borne diseases
}

\section{Introduction}

Three mosquito-borne diseases - malaria, dengue and filariasis - are among the major causes of illness and death in the tropical and subtropical regions of the world. In addition to the direct effects they exert by increasing premature mortality and morbidity, they are responsible for considerable economic wastage due to lost manpower and treatment costs, as well the direct costs of vector control. These effects constitute a serious impediment to economic development in countries in which these diseases are endemic.

\section{Malaria}

Malaria, the most serious vector-borne disease, is caused by four species of the genus Plasmodium. Human infections with the most virulent species, $P$. falciparum, may be fatal in non-immune individuals if untreated. A second species, P. vivax, is not usually lethal, but it may manifest itself or recur up to 3 years after the initial infection is acquired, because of the presence of dormant forms in the liver. These common parasites pose serious threats to public health in many tropical areas. The other species, $P$. malariae and $P$. ovale, are less common. The disease is transmitted by night-biting Anopheles mosquitoes and threatens $c$. $40 \%$ of the world's population. Each year it causes clinical illness, often very severe, in 300-500 million people and there are over a million deaths. Two scientific advances in the Second World War provided new chemical weapons to combat malaria and offered the hope that it may be eliminated as a major public health problem. The first was a range of drugs developed and tested by Allied scientists that effectively attacked the parasite in man. Two of these drugs, proguanil and chloroquine, were very effective, relatively cheap, and safe in therapeutic doses. The second scientific advance was the discovery of DDT. In 1955 the World Health Assembly advocated malaria eradication by country-wide 'residual spraying' campaigns involving the systematic application of the insecticide to the interior walls of houses to reduce the longevity of the anopheline vectors and to interrupt transmission of the disease.

The concept of a 'time-limited' eradication was stressed at the outset, as entomologists were aware of the likely development of resistance to DDT by the Anopheles vectors. This, and the recognition that resources dedicated to the eradication programmes could not be allocated indefinitely, were major incentives for speedy progress towards a final elimination of the disease. Initially, the DDT spraying campaigns achieved some notable successes. Transmission was interrupted in some countries and the incidence of disease was dramatically reduced in others. However, the goal of global eradication was not realised. Operational difficulties in many countries were reinforced by an increasing backlash against the widespread use of broad-spectrum organic insecticides, particularly DDT, by environmentalists. Moreover, the ongoing financial burdens of continued spraying as a malaria control measure could not be sustained. This was particularly true in countries that were in transition from colonial territories to independent nations. Insecticide resistance of mosquitoes was not a universal phenomenon, but it did develop in several countries and was sufficient to blunt the progress that might otherwise have been achieved. During the 1970s many of the early gains were lost and the worldwide malaria situation deteriorated to pre-spraying levels [1].

In parallel with the ongoing difficulties experienced by the DDT campaigns, a second, more insidious problem, emerged: the development of resistance of the parasites to antimalarial drugs. Within 5 years of the end of the war proguanil resistance was reported in Malaysia [2]. The first confirmed cases were from a relatively restricted area and the drug continued to be used as a suppressive, but its use for treatment declined. During the $1950 \mathrm{~s}$ there was no report of resistance to chloroquine and it was accepted as the drug of choice for both treatment and suppression of human malaria [3]. In 1961, chloroquine-resistant falciparum malaria was reported from Colombia [4] and the following year it was confirmed in Thailand [5]. During the next 10 years the prevalence of resistance spread to include most of South America and South-east Asia. Chloroquine resistance appeared during the 1970s in Africa and by the end of the 1980s it had spread to most malarious countries in the world [6]. Mefloquine was introduced in 1984, but within 5 years treatment failures were reported [7]. It has been suggested that malaria parasites may have the ability to develop resistance to any compound to which they are exposed [8]. The stage has now been reached at which no single 
compound can offer absolute protection against malaria in all areas of the world.

By the late 1960 s it was clear that global malaria eradication was a pipe dream, even though indigenous disease had been eradicated from Europe and Australia and there were notable reductions in incidence elsewhere. This was ultimately accepted by the World Health Organization (WHO) and the DDT campaigns have now faded into history. Anopheles moquitoes bite during the night, so the use of mosquito nets while sleeping can provide protection against the disease. In recent years there has been a renewed interest in this obvious and widely accepted method of malaria control. Bed nets impregnated with pyrethroid insecticides have been shown to give protection in some areas of Africa, the Americas, Asia and the Pacific and their use has been advocated by the WHO as part of the global strategy against malaria [9]. However, the long-term effectiveness of impregnated bed nets in reducing the incidence of malaria in any country has yet to be demonstrated and there may be implications for the development of childhood immunity in holoendemic areas [10].

Research on malaria vaccines is being undertaken in several countries, but none has been developed to the stage at which it can reliably prevent the disease in the field. It appears that it will be many years before an effective vaccine can be contemplated [11]. Development costs and ethical restraints concerning the use of human volunteers are currently restricting the development of new antimalarial drugs. Despite all that has been achieved in the fight against malaria during the last half century, it remains an intractable problem and continues to be a major scourge of humanity in the tropics.

\section{Dengue}

Dengue is the most important human viral disease transmitted by mosquitoes. Over half the world's population live in areas at risk of infection and up to 100 million cases of dengue fever occur annually [12]. The main vector is Aedes aegypti, a day-biting mosquito closely associated with human habitations, which breeds in artificial man-made containers. Other species, particularly Aedes albopictus, have also been incriminated as vectors in some areas. There are four different serotypes of dengue virus: DEN 1, 2, 3 and 4 . Infection with one serotype confers immunity to that virus, but there is no protective cross-immunity to the others. Dengue fever is a febrile acute illness with a range of clinical manifestations, including headache, joint pain and nausea, normally lasting for no more than a week. Dengue haemorrhagic fever is a more severe form distinguished by evidence of plasma leakage. Patients with dengue haemorrhagic fever can develop dengue shock syndrome with circulatory failure and shock that may prove fatal. There is no effective drug treatment for dengue and good nursing and clinical management are essential to prevent death in dengue haemorrhagic fever.

The incidence of dengue increased in South-east Asia soon after the Second World War and developed into a global pandemic that has intensified over the last 20 years. The reasons for this are not completely understood [13], but increased aircraft travel provides an ideal means of rapidly transporting the virus within infected travellers between cities in tropical countries. Dengue is primarily an urban disease of the tropics, where major demographic changes have taken place. Population movements from rural areas to cities have resulted in the proliferation of squatter settlements with inadequate housing and lack of reticulated water supplies. These conditions provide ideal breeding conditions for Aedes aegypti in water drums and other containers, such as old automobile tyres, which are common in many tropical urban areas. Insecticide application by truck-mounted or backpack ultra-lowvolume sprayers, has been widely used to control dengue vectors. Unfortunately, this approach is not very effective against adult mosquitoes. Larval control by application of insecticides in man-made containers is costly and usually does not result in reducing mosquito populations to levels that prevent transmission. Efforts to reduce such water collections by community clean up campaigns have been advocated, but are difficult to implement. It is particularly difficult to ensure that the actual breeding sites (rather than rubbish and garbage, which are not a source of mosquito breeding) are removed by such activities. There is no effective dengue vaccine at present and none is foreseen for the immediate future. Under these circumstances, the prospects for improving the global dengue situation are not encouraging.

Prospects for the control of other arthropod-borne virus diseases, such as yellow fever and Japanese B encephalitis, are equally uncertain, although these diseases are more restricted in distribution and effective vaccines are available. However, the occurrence of important non-human reservoirs complicates effective control of these viruses.

\section{Filariasis}

Human lymphatic filariasis is caused by infection with parasitic nematodes. In advanced infections it may cause elephantiasis, a painful and disfiguring swelling of the limbs and genitals. Bancroftian filariasis, caused by Wuchereria bancrofti, is the most widespread form of the disease with a geographical distribution extending through the tropics including parts of South America, Africa, Asia and the Pacific. Brugian filariasis, caused by Brugia malayi or B. timori, is restricted to south Asia. The larvae (microfilariae) 
circulate in the peripheral blood of the human host to infect mosquitoes. Their numbers in peripheral blood follow a periodicity that differs in different areas. Microfilariae of $W$. bancrofti exhibit nocturnal periodicity, except in Pacific islands where the disease is transmitted by day-biting mosquitoes.

Several different anopheline and culicine mosquitoes transmit filariasis in various parts of the world. Their control is hampered by the same kinds of difficulties which impede the control of malaria and dengue vectors. However, the transmission cycle from mosquitoes to man is relatively inefficient and filariasis is particularly susceptible to control efforts by drugs.

Diethylcarbamazine citrate (DEC) has been the mainstay of treatment for $>40$ years, while single-dose treatment with ivermectin can drastically reduce blood levels of microfilariae [14]. Single doses of DEC and ivermectin administered concurrently yield $>99 \%$ clearance of microfilariae for at least a year [15]. Single doses of albendazole, in combination with either ivermectin or DEC, seem equally effective [16]. These findings prompted the World Health Assembly in May 1997 to advocate the global elimination of lymphatic filariasis. The WHO will play a key co-ordinating role in assisting countries to develop their own elimination programmes with the objective of achieving active elimination campaigns in all countries by 2010 and the global elimination of the disease as a public health problem by 2020 .

A. W. SWEENEY, Pacific Regional Vector Borne Diseases Project, Secretariat of the Pacific Community, B.P. D5 98848 Noumea Cedex, New Caledonia (e-mail: sweeney@spc.org.nc)

\section{References}

1. Farid MA. The malaria programme - from euphoria to anarchy. World Health Forum 1980; 1: 8-22.

2. Edeson JFB, Field JW. Proguanil-resistant falciparum malaria in Malaya. $B M J$ 1950; 1: 147-151.

3. Coatney GR. Pitfalls in a discovery: the chronicle of chloroquine. Am J Trop Med Hyg 1963; 12: 121-128.

4. Moore DV, Lanier JE. Observations on two Plasmodium falciparum infections with an abnormal response to chloroquine. Am J Trop Med Hyg 1961; 10: 5-9.

5. Harinasuta T, Migasen S, Boonnag D. Chloroquine resistance in Plasmodium falciparum in Thailand. UNESCO first regional symposium on scientific knowledge of tropical parasites. Singapore, 1962: 148-153.

6. Payne D. Spread of chloroquine resistance in Plasmodium falciparum. Parasitol Today 1987; 3: 241-246.

7. Karwacki JJ, Webster HK, Limsongwong N, Shanks GD. Two cases of mefloquine resistant malaria in Thailand. Trans $R$ Soc Trop Med Hyg 1989; 83: 152-153.

8. Wernsdorfer WH. The development and spread of drugresistant malaria. Parasitol Today 1991; 7: 297-303.

9. Schreck CE, Self LS. Bed nets that kill mosquitos. World Health Forum 1985; 6: 342-344.

10. Snow RW, Omumbo JA, Lowe B et al. Relation between severe malaria morbidity in children and level of Plasmodium falciparum transmission in Africa. Lancet 1997; 349: 1650-1654.

11. Mendis KN. Malaria vaccine research - a game of chess. In: Targett GAT (ed) Malaria: waiting for the vaccine (London School of Hygiene and Tropical Medicine First Annual Public Health Forum.) Chichester, John Wiley and Sons. 1991: $183-196$.

12. Rigau-Perez JG, Clark GG, Gubler DJ, Reiter P, Sanders EJ, Vorndam AV. Dengue and dengue haemorrhagic fever. Lancet 1998; 352: $971-977$.

13. Gubler DJ, Clark GG. Dengue/dengue hemorrhagic fever: the emergence of a global health problem. Emerg Infect Dis 1995; 1: $55-57$.

14. Chodakewitz J. Ivermectin and lymphatic filariasis: a clinical update. Parasitol Today 1995; 11: 233-235.

15. Ottesen EA, Ramachandran CP. Lymphatic filariasis infection and disease: control strategies. Parasitol Today 1995; 11: 129-131.

16. Ismail MM, Jayakody RL, Weil GJ et al. Efficacy of single dose combinations of albendazole, ivermectin and diethylcarbamazine for treatment of bancroftian filariasis. Trans $R$ Soc Trop Med Hyg 1998; 92: 94-97. 